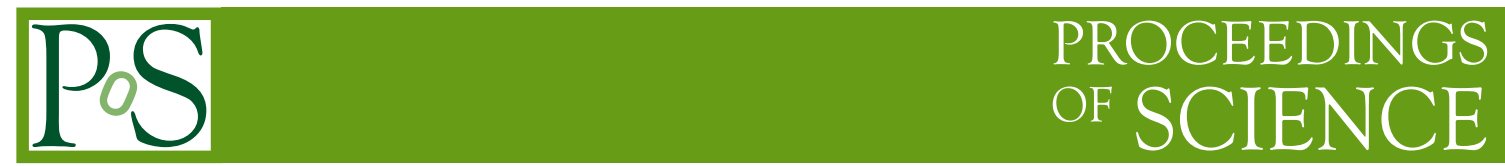

\title{
Search for Heavy Stable Charged Particles in CMS
}

\author{
Małgorzata Kazana * \\ For the CMS Collaboration \\ The Andrzej Sottan Institute for Nuclear Studies, Poland \\ E-mail: Malgorzata.Kazana@fuw.edu.pl
}

The study of the detection sensitivity to a heavy stable charged particle (HSCP) at the CMS detector is presented. The HSCP hypothetical particles arise in many models including SUSY and extra dimensions. The CMS detector is able to measure their velocity and specific ionization. This redundancy allows for substantial background reduction and HSCP mass determination. These studies are based on detailed detector simulation, including all Standard Model backgrounds. Particular emphasis is given to possible early discoveries, i.e. with $1 \mathrm{fb}^{-1}$ and $100 \mathrm{pb}^{-1}$ or less for proton-proton collisions at center of mass energy of $14 \mathrm{TeV}$.

European Physical Society Europhysics Conference on High Energy Physics, EPS-HEP 2009,

July 16 - 222009

Krakow, Poland

* Speaker. 


\section{Introduction}

Extensions of the Standard Model predict new charged particles with masses above $100 \mathrm{GeV}$. Under certain circumstances they can be semi-stable. While a life time (c $\tau$ ) of the HSCP is a few meters, the particle cross the detector like muons. Such long-lived particles are predicted by a specific supersymmetry and extra dimensions models. As benchmark models for the purpose of the CMS analysis[1] are taken: Gauge Mediated Supersymmetry Breaking with long-lived staus $\left(m_{\tilde{\tau}}=156,247 \mathrm{GeV}\right)$, Extra Dimensions with long-lived KK states $\left(m_{\tau_{K K}}=300 \mathrm{GeV}\right)$ and Split SUSY with long-lived R-hadrons (gluino: $m_{\widetilde{g_{1}}}=200-1500 \mathrm{GeV}$, stop: $m_{\widetilde{t_{1}}}=130-800 \mathrm{GeV}$ ).

Typically, the HSCP is slower $(\beta<c)$ than Standard Model (SM) particles produced at the same p-p collision and it is detected with a measurable time delay inside the detector. The ionization energy loss $(d E / d x)$ for a heavy charged particle is higher than for a SM one. The time delay and the $d E / d x$ provide two independent estimates of the HSCP velocity. The information about the particle's velocity combined with the standard momentum measurement allows to determine the HSCP's mass, $m=p \sqrt{1 / \beta^{2}-1}$.

\section{Estimates of particle velocity}

In Drift Tubes (DT) used by the CMS[2] barrel Muon System, the position of a charged particle track is measured from the drift time of the ionization electrons created along the particle's path. The DTs are synchronized in a way that a relativistic muon produced in the p-p interaction gives an aligned pattern of hits. For a slower particle, the reconstructed hits in each tube are shifted with respect to their real position. The hits form a zig-zag pattern with an offset proportional to the particle delay. From the position of these hits, it is possible to compute the particle time delay with respect to a real muon, the total time of flight and its velocity. The only requirement is that at least three hits are found in one of the four muon stations and that the track is registered by a perpendicular layer in the same muon station.

An initial part of the charged particle track is reconstructed in the CMS Silicon Strip Tracker (Tk), which consists of over $200 \mathrm{~m}^{2}$ of silicon sensor planes with almost 10 million readout channels. Measurements are made in sequential layers of the tracker and recorded as RecHit objects with associated position and a cluster charge, which is define as the sum of the heights of the signals on the strips that form a cluster. The cluster charge is proportional to the amount of energy released by the charged particle crossing the silicon module. The $d E / d x$ is computed from the collected signals once the normalization factor is determined directly from data using low momentum protons and kaons. Since the energy losses depends on the velocity of the particle according to a $1 / \beta^{2}$ dependence for $0.1<\beta<0.8 \div 0.9$, the value of $\beta$ can be derived.

\section{Online and offline selections}

Events with HSCPs will be triggered by standard trigger paths although the algorithms of the instrumental and software level trigger were not design for such slow particles. Slow HSCPs can be reconstructed in different bunch crossings or can fail reconstruction at all. The best results are achieved with the muon trigger if the HSCP is self-triggered or if SM muons which are present in 
the event with the HSCP give a trigger. Many models predict a large missing transverse energy (MET) and jets from cascade decays in which a HSCP is produced, therefore the JET/MET paths are also used. The final trigger efficiency is found to be about $99 \%$ for GMSB HSCPs, $80 \%$ for KK tau and $40-95 \%$ for the R-hadrons.

The background for HSCPs consists of wrongly measured muons which can come from any SM processes or cosmic muons which cross the center of the detector. 150 millions of simulated p$\mathrm{p}$ collision events and 10 millions of cosmic muons were used for background evaluation. Samples with fully simulated response of the detector were re-weighted in order to correspond to $1 \mathrm{fb}^{-1}$ of data and include, among others, $\mathrm{W}+\mathrm{jet}, \mathrm{Z}+\mathrm{jet}$, $\mathrm{tt}+\mathrm{jet}$ samples and Drell-Yan, $\mathrm{Z}, \mathrm{W}$, bottomonia, charmonia processes which are rich in muons.

The offline selection is based on velocity $(\beta)$ measurements made by the DT muon system and the tracker, with following requirements. Candidates are selected from muons with momentum $p_{T}>30 \mathrm{GeV}$ or tracks with $p_{T}>30 \mathrm{GeV}$ and $\beta_{T k}<0.9$, number of $d E / d x$ hits $\geq 9$ and $\chi_{T k}^{2}<5$. A signal region is defined in a parameter space of $\beta_{D T}$ and $\beta_{T k}$ for muons associated with tracks $\left(\Delta\left(1 / p_{T}\right)<0.005 / \mathrm{GeV}\right.$ and $\left.\Delta R<0.1\right)$ if $\beta_{D T}<0.8, \sigma\left(1 / \beta_{D T}\right)<0.1$ and $\beta_{T k}<0.8$. The mass of a candidate (average from $\beta_{D T}$ and $\beta_{T k}$ ) has to be larger than $100 \mathrm{GeV}$. The number of expected background events for a luminosity of $1 \mathrm{fb}^{-1}$, using this selection cuts above, is much less than 1. The final efficiency of the signal is dominated by the trigger efficiency, the acceptance of the DT detector (barrel region) and the $\beta$ spectrum in a given model. The overall efficiency for the selection is between $6 \%$ and $30 \%$.

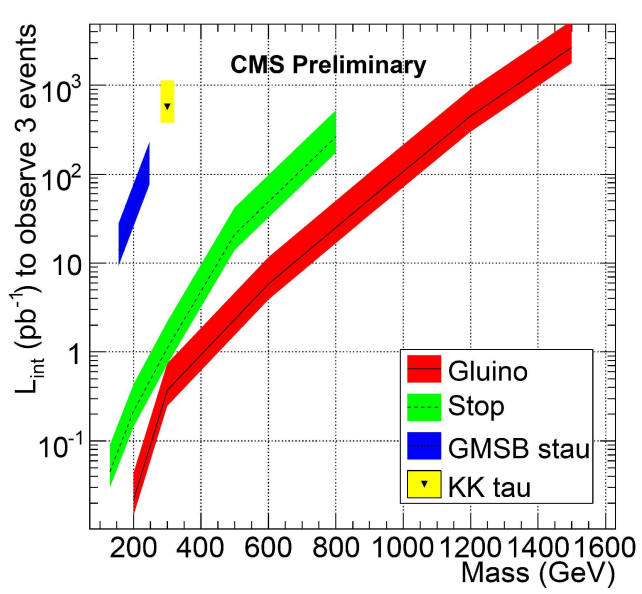

Figure 1: The CMS discovery reach of HSCPs at $14 T e V$ with Drift Tubes and Tracker as an integrated luminosity needed for a discovery with 3 events for four signal models as a function of the HSCP's mass.

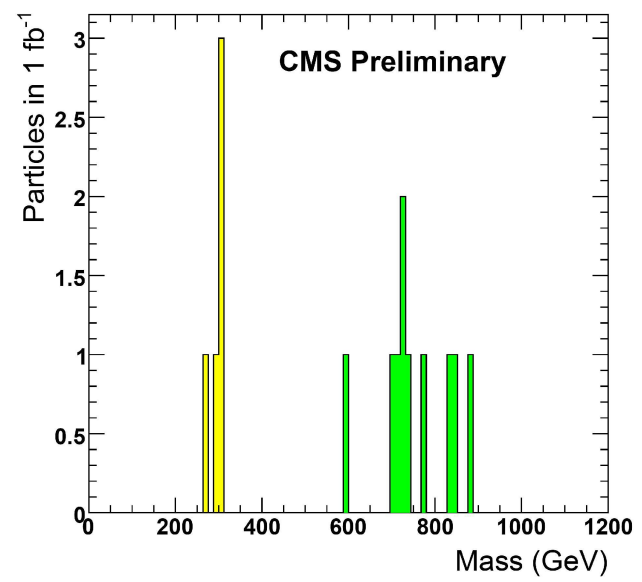

Figure 2: The reconstructed mass distribution with luminosity of $1 \mathrm{fb}^{-1}$ for two signal samples with masses of $300 \mathrm{GeV}\left(\tau_{K K}\right)$ and $800 \mathrm{GeV}\left(\widetilde{t_{1}}\right)$.

\section{Results}

A discovery in the signal region can be claimed if at least 3 events are observed when no background events are expected. The luminosity needed for the HSCP's discovery with Drift Tubes and Tracker is shown in Figure 1. Systematic uncertainties are included and marked as error bars. 
With a luminosity of $100 \mathrm{pb}^{-1}$, the existence of the stau can be verified up to a mass of $250 \mathrm{GeV}$. The discovery limits with $100 \mathrm{pb}^{-1}$ for R-hadrons reach a mass of $700 \mathrm{GeV}$ for charged stop and $1 \mathrm{TeV}$ for neutral gluino. For a newly discovered particle, its mass will be determined. The expected mass distributions for two models with HSCP are presented in Figure 2. In case of an excess of events in the signal region more sophisticated analyses including standalone studies with DT and Tracker only are planned.

\section{References}

[1] G. L. Bayatian et al. [CMS Collaboration], "Search for Heavy Stable Charged Particles with 100pb and $1 \mathrm{fb}^{-1}$ in the CMS experiment", CMS-EXO-08-003;

[2] G. L. Bayatian et al. [CMS Collaboration], "CMS physics: Technical design report”, CMS-TDR-008-1; 